\title{
Biomedical Applications of Supramolecular Materials in the Controllable Delivery of Steroids
}

\author{
Yun Hao ${ }^{1,2}$, Feiyi Zhang ${ }^{3,4}$, Shanshan $\mathrm{Mo}^{4}$, Jinming Zhao ${ }^{1,2}$, Xiangdong Wang ${ }^{1,2}$, \\ Yan Zhao ${ }^{1,2 *}$ and Luo Zhang ${ }^{1,2,5 *}$
}

${ }^{1}$ Department of Otolaryngology Head and Neck Surgery, Department of Allergy, Beijing TongRen Hospital, Capital Medical University, Beijing, China, ${ }^{2}$ Beijing Key Laboratory of Nasal Diseases, Beijing Institute of Otolaryngology, Beijing, China, ${ }^{3}$ Institute for Advanced Materials, Jiangsu University, Zhenjiang, China, ${ }^{4}$ State Key Laboratory of Medical Molecular Biology, Institute of Basic Medical Sciences Chinese Academy of Medical Sciences, School of Basic Medicine Peking Union Medical College, Beijing, China, ${ }^{5}$ Chinese Academy of Medical Sciences and Peking Union Medical College, Beijing, China

OPEN ACCESS

Edited by:

Xiaoguang Wang,

The Ohio State University,

United States

Reviewed by:

Min Li,

Institute of High Energy Physics (CAS),

China

Yibing Wang,

East China University of Science and

Technology, China

*Correspondence:

Luo Zhang

dr.luozhang@139.com

Yan Zhao

zhaoyanray@126.com

Specialty section:

This article was submitted to

Nanobiotechnology,

a section of the journal

Frontiers in Molecular Biosciences

Received: 26 April 2021

Accepted: 13 July 2021

Published: 23 July 2021

Citation:

Hao Y, Zhang F, Mo S, Zhao J, Wang $X$, Zhao $Y$ and Zhang $L$ (2021)

Biomedical Applications of

Supramolecular Materials in the

Controllable Delivery of Steroids.

Front. Mol. Biosci. 8:700712.

doi: $10.3389 /$ fmolb.2021.700712
Glucocorticoids are a class of steroid hormones secreted from the adrenal glands. The strong anti-inflammatory effects make it be one of the most popular and versatile drugs available to treat chronic inflammatory diseases. Additionally, supramolecular materials have been widely exploited in drug delivery, due to their biocompatibility, tunability, and predictability. Thus, steroid-based supramolecular materials and the release of steroids have been applied in the treatment of inflammatory diseases. This mini-review summarized recent advances in supramolecular materials loaded with glucocorticoid drugs in terms of hydrophobic interactions, electrostatic interactions, hydrogen bonding, and $\pi-\pi$ stackings. We also discussed and prospected the application of the glucocorticoid drugs-based supramolecular system on chronic rhinosinusitis, multifactorial inflammatory disease of the nasal and paranasal sinuses mucosal membranes. Overall, supramolecular materials can provide an alternative to traditional materials as a novel delivery platform in clinical practice.

Keywords: steroids, supramolecular materials, interaction, biomedical application, chronic rhinosinusitis

\section{INTRODUCTION}

Glucocorticoids belong to the family of cholesterol-derived hormones produced by the adrenal glands. They have been discovered and applied into clinical medicine in the 1940s (Vandewalle et al., 2018) and can be administered orally, intravenously, or topically (Luhder and Reichardt, 2017). Considering their strong and efficient anti-inflammatory effects, glucocorticoids has been generally introduced to the treatment of rheumatoid arthritis (Aletaha and Smolen, 2018), asthma (Barnes, 2011), atopic dermatitis (Mayba and Gooderham, 2017), allergic rhinitis (Wheatley and Togias, 2015) and chronic rhinosinusitis (Hopkins, 2019). However, the side effects of glucocorticoids cannot be ignored. They can arise hypothalamic-pituitary-adrenal suppression-related complications such as hyperglycemia, aseptic necrosis of the femoral head, negative calcium balance, mood disorders, and Cushing's syndrome (Chotiyarnwong and McCloskey, 2020). It is urgent to develop a novel therapeutic regimen for steroids delivery and release.

Traditional chemistry used in the synthesis and application of glucocorticoids focuses on the covalent bond; however, supramolecular chemistry mainly examines the weaker and reversible noncovalent interactions such as hydrophobic, electrostatic, hydrogen bonding, and $\pi-\pi$ stackings (Mendes et al., 2013). In general, molecules self-assembled by non-covalent interactions in specific solvent and formed supramolecular materials (Zhang et al., 2020a). Supramolecular materials have 
biocompatibility, tunability, and predictability (Zhou et al., 2017; Yu et al., 2020). Thanks to the advantages of supramolecular materials, significant advances have been made in drug delivery. Glucocorticoid drugs have many characteristics for loading onto supramolecular materials. Firstly, their typical hydrophobic structure promotes the combination with amphiphilic supramolecular materials. Secondly, the incorporation of anionic groups assists in combining with materials through electrostatic interactions. Lastly, their ordered structure and abundant $\pi$ electrons indicate the existence of hydrogen bonds and $\pi-\pi$ stackings.

In this review, we outlined the recent progress on basic theories and biomedical applications in supramolecular materials with glucocorticoid drugs via four kinds of interactions including hydrophobic interaction, electrostatic interaction, hydrogen bond, and $\pi-\pi$ stackings. We hope our review would provide new ideas for the application of steroidsbased supramolecular materials in the treatment of more diseases.

\section{HYDROPHOBIC INTERACTIONS}

Hydrophobic interaction is a tendency of nonpolar substances to aggregate in an aqueous solution, which increases hydrogen bonds between water molecules and decreases the area of contact between water and nonpolar molecules. Distinct from electrostatic interactions and hydrogen bonds, hydrophobic interactions can be elucidated as a thermodynamic effect with changes in free energy, entropy, enthalpy, and heat capacity rather than one of the fundamental types of molecular interactions (Shen et al., 2017; Yadav et al., 2020). When hydrophobic groups of molecules contact with water molecules, the entropic effect leads to a rearrangement of water molecules. Researchers have increasingly focused more attention on biomaterials to make the best use of hydrophobic interactions. It is commonly observed that without applying amphiphilic materials or combining with hydrophilic molecules, hydrophobic drugs alone fail to self-assemble to nanostructures (Wang et al., 2012). Therefore, some bonds such as disulfide, thioether, ester bonds, or amphiphilic materials were added to the hydrophobic drugs to selfassemble into nanostructures in aqueous solutions (Wang et al., 2014).

It has been demonstrated that the glucocorticoids could attenuate inflammations in acute lung injury (Tu et al., 2017), arthritis (Aletaha and Smolen, 2018), and atherosclerosis (van der Sluis and Hoekstra, 2020). Compared to normal cells, inflammatory cells tend to overexpress reactive oxygen species (ROS), accompanied by the inflammatory process. Hence, ROSresponsive nanoparticles have been introduced as targets for precise drug release treatment in inflammatory diseases. Ma et al. (Ma et al., 2020) reported that a ROS-responsive linkage was used to bridge the prednisolone and two-photon fluorophore (TP), constituting a diagnosis-therapy compound named TPP. Adding TP to prednisolone promoted the hydrophobic interaction of steroids obviously and provided a dimensional location for inflammation. The compound TPP was then packaged with an amphipathic diblock copolymer poly (2methacryloyltoxyethyl phosphorylcholine)-poly (2-methylthio ethanol methacrylate) (PMPC-PMEMA) via hydrophobic interaction. The compound TPP and amphipathic copolymer PMPC-PMEMA were dissolved in the DMSO and methanol solution with phosphate-buffered saline (PBS) was added dropwise, stirred, and filtered. Afterward, the self-assembled form was obtained. With PMPC serving as a hydrophilic segment and PMEMA as a hydrophobic block, PMPCPMEMA exhibited great superiority of protein adsorption resistance, biocompatibility, and responsiveness to ROS. The results showed that the particle size increased and prednisolone was released with the increased concentration of $\mathrm{H}_{2} \mathrm{O}_{2}$, an oxidizer that induces reactive ROS formation (Ogawa et al., 2004). Prednisolone was slowly released without the stimulation of $\mathrm{H}_{2} \mathrm{O}_{2}$, while it showed rapid release under the $\mathrm{H}_{2} \mathrm{O}_{2}$ effect. After $48 \mathrm{~h}$, there was approximately $89 \%$ of prednisolone delivery. After being treated with $\mathrm{H}_{2} \mathrm{O}_{2}$ for $4 \mathrm{~h}$, noncompact micelles started to aggregate due to the hydrophobic interaction of TP, suggesting the outstanding reversibility of these supramolecular materials (Figure 1A).

In recent years, some in vivo disease models have been established to evaluate the effectiveness of the supramolecular materials loaded with glucocorticoids. They found that the supramolecular materials loaded with glucocorticoids treatment could significantly inhibit the pulmonary edema in the acute lung injury mice model than saline group. In addition, the supramolecular materials loaded with glucocorticoids treatment also exhibited a more detumescence effect than the untreated group in the collagen-induced arthritic joints model. Furthermore, it could strongly reduce oxidized low-density lipoprotein uptake and inhibit foam cell formation for atherosclerosis in vitro and in vivo. The outstanding theragnostic of inflammation for the glucocorticoid drug delivery system has been proved in the treatment of acute lung injury, arthritis, and atherosclerosis (Ma et al., 2020).

Moreover, Chung et al. Chung et al. (2020) reported the synthesis of another supramolecular material combined with steroids. Poly (ethylene glycol) (PEG) played a hydrophilic role, while rosmarinic acid (RA), an anti-inflammatory and anticancer component, played a strong hydrophobic role. They were prepared and self-assembled, relying on hydrophobic interactions when dexamethasone was incorporated into the solution. Steroid-based hydrogel treatment could recover colon length dose-dependently in the dextran sulfate sodium salt (DSS) induced colitis mice model (Chung et al., 2020).

Generally, hydrophobic interaction is a common non-covalent interaction widely applied to various fields. As for steroids, a kind of prominent hydrophobic drug, hydrophobic interaction with supramolecular materials might be a trend in the future of drug delivery (Figure 2; Table 1).

\section{ELECTROSTATIC INTERACTIONS}

Electrostatic interactions are long-range non-covalent interactions between two charged ions or molecules, occurring 


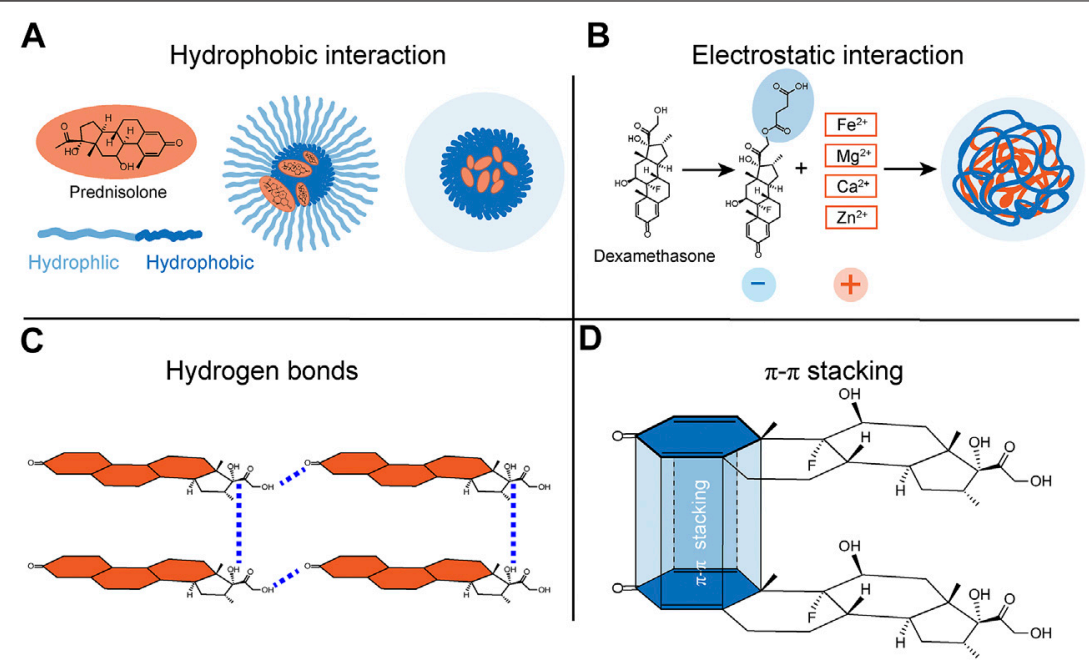

FIGURE 1 | Schematic representations of possible interactions between supramolecular materials and steroids. The supramolecular materials bind to steroids possibly via (A) hydrophobic interactions, (B) electrostatic interactions, (C) hydrogen bonds, and (D) $\pi$ - $\pi$ stacking interactions.

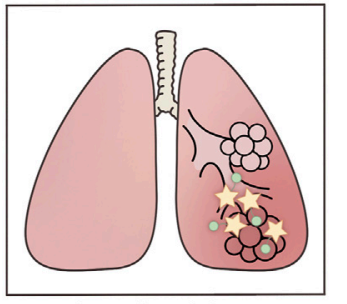

Acute lung injury

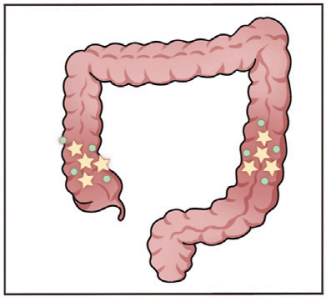

Inflammatory bowel disease

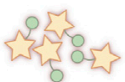

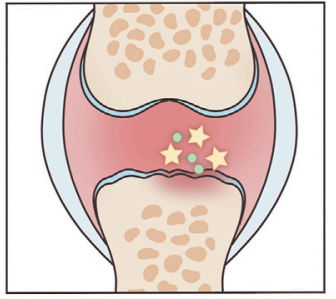

Arthritis

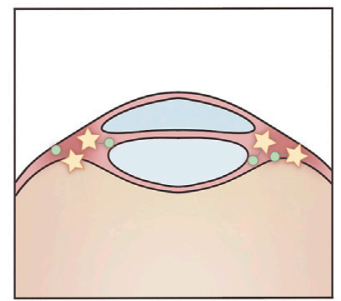

Non-infection uveitis

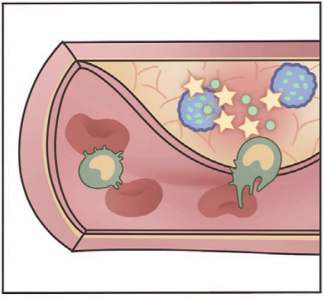

Atherosclerosis

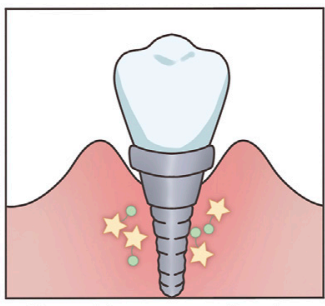

Dental impact application

Steroid Supramoleculer material

FIGURE 2 | The biomedical applications of the glucocorticoid drug delivery systems. Glucocorticoid drugs based supramolecular systems have been widely used in acute lung injury, arthritis, atherosclerosis, inflammatory bowel disease, non-infection uveitis, and dental impact application.

when ions or molecules with opposite charges attract each other (Schneider, 2009). Changes in the $\mathrm{pH}$ and ionic strength of the solution can moderate the forces of electrostatic interactions (Shen et al., 2017). Electrostatic interactions have been used widely in some positively or negatively charged parts of the body. For instance, drugs encapsulated in cationic carriers could rapidly penetrate negatively charged cartilage (Bajpayee and Grodzinsky, 2017). Another drug was provided with a positive charge to bond with the negatively charged bladder mucosa to achieve sustained drug release (Guo et al., 2017).
Contrary to the cartilage and bladder, the drug delivery problem that should be overcome in mucosal tissues is rapid removal by the mucus. Drugs can be trapped in mucosa via electrostatic or hydrophobic interactions (Dunnhaupt et al., 2015). A high density of positive and negative surface charges facilitates drug delivery through the mucosal layer by minimizing the electrostatic interactions with mucus.

As a typical case of the mucosa, in the field of non-infectious uveitis, Zhou et al. Zhou et al. (2018) designed and synthesized steroidal drug-based supramolecular hydrogels by electrostatic 
TABLE1 | Features and medical applications of different supramolecular interactions used for the delivery of steroids.

\begin{tabular}{ll} 
Interactions & \\
\hline Hydrophobic interaction & Ma et al. (2020) \\
& Chung et al. (2020) \\
Electrostatic interactions & Zhou et al. (2018) \\
& Wu et al. (2017) \\
Hydrogen bond & Fraile et al. (2016) \\
& Wu et al. (2017) \\
$\pi-\pi$ stacking interaction & Jung et al. (2015) \\
& Wu et al. (2017)
\end{tabular}

Medical applications

Acute and chronic inflammation

Acute inflammatory bowel disease

Non-infectious uveitis

Non-infectious uveitis

Posterior segment ocular diseases

Non-infectious uveitis

Dental implant applications

Non-infectious uveitis
References (PMID)

PMID: 32379416

PMID: 32449857

PMID: 29803956

PMID: 28501709

PMID: 27594212

PMID: 28501709

PMID: 25909563

PMID: 28501709 interactions and metal coordination. Firstly, a carboxylic acid group was added to dexamethasone to enhance electronegativity. Succinate dexamethasone (Dex-SA) was suspended in PBS, followed by incorporating $\mathrm{Na}_{2} \mathrm{CO}_{3}$ solution to provide a transparent solution. Next, due to the incorporation of various cations $\mathrm{Mg}^{2+}, \mathrm{Ca}^{2+}, \mathrm{Zn}^{2+}, \mathrm{Fe}^{2+}, \mathrm{Cu}^{2+}$, and $\left.\mathrm{NH}_{4}{ }^{+}\right)$, Dex-SA selfassembled to form steroidal supramolecular hydrogels. Besides, the results showed distinct gelation ability among cations in the order of $\mathrm{Mg}^{2+}>\mathrm{Ca}^{2+}>\mathrm{Zn}^{2+} \approx \mathrm{Fe}^{2+>} \mathrm{NH}_{4}^{+}$. Divalent cations exhibited strong gelation ability due to two sites coordinating with a carboxylic acid. The ability of various divalent cations to induce gelation might be affected by the radii of cations. Cations with smaller radii lead to a stronger ability for gelation. Among the above cations, $\mathrm{Ca}^{2+}$ ions are most widely distributed in humans; therefore, attention has been focused on the synthesis and application of the $\mathrm{Ca}^{2+}$-Dex-SA supramolecular hydrogel. This $\mathrm{Ca}^{2+}$-Dex-SA supramolecular hydrogel exhibited good drug delivery ability modulated by changes in the $\mathrm{Ca}^{2+}$ concentration. Within $6 \mathrm{~h}$, almost all Dex-SA and dexamethasone were completely released from the hydrogel. In addition, $\mathrm{Ca}^{2+}$-DexSA supramolecular hydrogel showed good reversibility upon large-amplitude oscillatory shear cycle. With an increase in $\mathrm{Ca}^{2+}$ concentration, the drug release rate slowed down, suggesting that the supramolecular material is $\mathrm{Ca}^{2+}$ responsive.

In addition, steroid-based supramolecular materials might be very effective for inflammation of non-infection uveitis. The noninfection uveitis rabbit model was induced by the carrageenan injection to increase epithelial thickness and the appearance of a horny superficial layer on the corneal surface. The triamcinolone acetonide incorporated into micellar solution treatment could improve the histological architecture of the cornea and nearly normal epithelial pattern for the corneal tissue (Safwat et al., 2020). With the rapid development of nanotechnology, the more and more extensive biological application of steroid-based supramolecular materials has been achieved (Figure 2; Table 1). And the widespread distribution of $\mathrm{Ca}^{2+}$ ions in the human body certainly provides us with a good prospect for further applications of supramolecular materials via electrostatic interactions (Figure 1B).

\section{HYDROGEN BONDS}

A hydrogen bond is an electrostatic interaction between the hydrogen with a partial positive charge atom or atomic group with a partial negative charge, where there is evidence of a bond formation. The electronegative atom directly connected with hydrogen bond by covalent bond is the hydrogen bond donor, while the hydrogen bond acceptor is the other electronegative atom which is not covalently attached to the hydrogen but participating in the formation of hydrogen bond. It is stronger than a van der Waals bond and weaker than fully covalent or electrostatic bonds. Of all the noncovalent bonds, a hydrogen bond is the one with the most pronounced directionality (Schneider, 2009). Some research teams synthesized layer-by-layer films employing hydrogen bonds between biologically compatible poly (acrylic acid) and a biodegradable block copolymer micelle to fully utilize the sensitive and directional characteristics of hydrogen bonds (Kim et al., 2008).

Concerning the use of hydrogen bonds on steroids, researchers dissolved dexamethasone and $\mathrm{Ca}^{2+}$ in distilled water to form dexamethasone supramolecular hydrogel. X-ray diffraction (XRD) analysis of dexamethasone supramolecular hydrogel suggested that the ordered structure exhibited a greater tendency to form hydrogen bonds and $\pi-\pi$ stackings ( $\mathrm{Wu}$ et al., 2017). The study speculation was reasonable, but no experimental verification was carried out to prove the formation of hydrogen bonds. The release of dexamethasone from hydrogel was categorized into a rapid release within the first $24 \mathrm{~h}$ and a sustained release in the subsequent $48 \mathrm{~h}$. They also found that dexamethasone-based supramolecular hydrogel injection could significantly efficacy on the suppression of the uveal inflammatory response, while the saline group exhibited a severe inflammatory response in the anterior chamber accompanied by a large amount of purulent exudate ( $\mathrm{Wu}$ et al., 2017) (Figure 2; Table 1).

Fraile et al. validated the formation of hydrogen bonds on dexamethasone through Fourier transform infrared (FT-IR) spectra and nuclear magnetic resonance (Fraile et al., 2016). They used laponite as a carrier for the controlled delivery of dexamethasone via hydrogen bonds. Dexamethasone was dissolved in ethanol or acetone, and laponite was added to this solution and stirred at room temperature; the solution self-assembled to form transparent colloidal dispersions. After removing the solvent, the whole amount of dexamethasone was deposited on the solid laponite. It is known that hydrogen bonds shift the X-H stretching frequency to a lower energy level (Feldblum and Arkin, 2014). The spectra results showed that, compared to the laponite alone group, the same $\mathrm{C}=\mathrm{O}$ and $\mathrm{C}=\mathrm{C}$ bands were slightly enlarged in the dexamethasone/laponite group, indicating that hydrogen bonds had formed in 
dexamethasone (Figure 1C). Furthermore, the carbonyl groups suffered a significant downfield shift in the ${ }^{1} \mathrm{H}$ spectrum due to the decline in electronic density when accepting a hydrogen bond. The release of dexamethasone was based on the equilibrium between the physiosorbed and the dissolved forms of dexamethasone. The release of dexamethasone increased when its concentration was low. Self-assembled through hydrogen bonds, supramolecular materials loaded with steroid drugs will improve their responsiveness, biocompatibility, and tunability.

\section{$\pi-\pi$ STACKING INTERACTIONS}

A $\pi-\pi$ stacking interaction refers to a type of noncovalent interaction involving unsaturated hydrocarbon predominantly containing $\pi$ bonds (Wheeler, 2013). The $\pi-\pi$ interaction can be categorized into T-shaped (edge-to-face stacked) and F-shaped (offset stacked and face-to-surface stacked), depending on the three-dimensional morphology of aromatic group interactions (Butterfield et al., 2002; Sinnokrot et al., 2002; Yu et al., 2020).

It was reported that supramolecular nanomaterials made of graphene had a honeycomb lattice framework where every carbon atom has its $\pi$ electrons (Shim et al., 2017). The high density of $\pi$ electrons makes it possible to use graphene-based nanomaterials for drug delivery via $\pi-\pi$ interactions (Shim et al., 2016). Thus, there are quite a few aromatic drugs containing $\pi$ electrons loaded on the graphene surface, suggesting that $\pi-\pi$ stacking interactions play an outstanding role in designing chemical drug delivery systems (Lee et al., 2011).

In a study on multi-pass caliber-rolled $\mathrm{Ti}$ alloy of $\mathrm{Ti}_{3} \mathrm{Nb}_{3} \mathrm{Zr}$ (MPCR-TNZ) for dental implant applications, the high mechanical strength MPCR-TNZ was surface-modified with graphene oxide and loaded with the osteogenic drug dexamethasone. The weak strength of $\pi-\pi$ interaction makes accurate measurements difficult in experiments (Zhuang et al., 2019). Considering the ordered structure in graphene and a large number of $\pi$ electrons in graphene and dexamethasone, $\pi-\pi$ stacking interactions could easily be speculated (Jung et al., 2015) (Figure 1D). In vitro, $10 \%$ of a total load of dexamethasone was released for a week, suggesting a long-term anti-inflammatory effect. The MPCR-TNZ loaded with dexamethasone showed facilitated differentiation of progenitor cells into osteoblasts compared to MPCR-TNZ material alone. Also, the dexamethasone-MPCR-TNZ group exhibited a significant amelioration in calcium-nodule formation and remarkable osteocalcin expression (Figure 2; Table 1).

Additionally, a study speculated the formation of $\pi-\pi$ stacking and hydrogen bond interactions in dexamethasone supramolecular hydrogel (Wu et al., 2017). Although the application of $\pi-\pi$ stacking interactions loaded with steroid drugs is relatively limited, $\pi-\pi$ stacking interaction still has a good application prospect because of the ordered structure of steroids.

\section{CONCLUSIONS AND PERSPECTIVES}

This review discussed and summarized the hydrophobic interactions, electrostatic interactions, hydrogen bonds, and $\pi-\pi$ stackings between supramolecular materials with steroid drugs and their biomedical applications. Understanding the basic theory of their hydrophobic groups, ordered structure and abundant $\pi$ electrons clearly will help us to make more effective supramolecular materials-based steroid drugs.

At present, the steroid-based supramolecular systems have been widely used in acute lung injury, arthritis, atherosclerosis, inflammatory bowel disease, non-infection uveitis, and dental impact application. There are still some diseases that require better use of this steroid-based supramolecular system to develop new treatment strategies. For example, chronic rhinosinusitis (CRS) is a chronic heterogeneous disease that encompasses complex multifactorial inflammatory conditions of the nasal and paranasal sinuses mucosal membranes. According to current guidelines, intranasal glucocorticoids, systematic glucocorticoids, and functional endoscopic sinus surgery (FESS) are the principal therapeutic approaches for CRS (Fokkens et al., 2019). While only surgery may not control the persistent inflammation, it is essential to control postoperative inflammation and scarring, and these postoperative patients thus require ongoing oral or topical corticosteroids to reduce the inflammatory burden in the sinuses (Kohanski et al., 2018). In the recent decade, a new type of bioabsorbable steroid-eluting stent that engineered from polylactide-co-glycolide (PLGA) impregnated with steroid medication has emerged for CRS treatment (Murr et al., 2011; Han et al., 2014; Janisiewicz and Lee, 2015; Smith et al., 2016; Kern et al., 2018). The steroids were combined with PLGA materials using a physical coating method via dipping, spraying, brushing, or the layer-by-layer (LBL) assembly technology (Rykowska et al., 2020). However, there are some limitations in the application of PLGA. For example, the acidic by-products of PLGA can cause inflammation in the surrounding tissues. Additionally, the drugeluting coronary stents reported that biodegradable stents (including PLGA stents) were associated with a higher stent thrombosis rate (Cassese et al., 2016). Also, an in vitro and in vivo study found prominent inflammation, eosinophil infiltration, and fibrotic tissue after stent implantation, indicating a prominent foreign-body reaction (Nikoubashman et al., 2018). Considering these limitations, specific research is necessary on promising materials to be utilized as a steroid-eluting stent. Owing to the properties in ordered superstructures, high responsiveness, biocompatibility, reversibility, tunability, and predictability, the combination of steroids and supramolecular materials may be expected to achieve sustained release as well as reduce the complications. Utilizing the responsiveness and reversibility, the self-assembly/disassembly processes of steroids can be controlled under chemical, physical, and biological stimuli.

Although supramolecular materials can be optimized to become a new generation of steroid drug delivery systems by skillfully adjusting these interactions. Nevertheless, several challenges limit the application of supramolecular materials containing steroids. Firstly, in most cases, the drug release time of supramolecular materials is less than that of macromolecular polymers. To achieve a long-lasting antiinflammatory effect, prolonging drug release time will undoubtedly have a significant impact on optimizing supramolecular materials. The combination of supramolecular materials and macromolecular polymers might become a new drug delivery platform. Secondly, efforts are needed to develop bio-signaling-based release 
mechanisms. In tumors and some inflammatory diseases (Zhang et al., 2020b), the $\mathrm{pH}$ (Deirram et al., 2019), temperature (Zavgorodnya et al., 2017), redox potential (Raza et al., 2018), and even specific overexpressed proteins (Shigemitsu et al., 2020) could serve as stimulations to trigger the drug release of supramolecular materials. Besides, other steroid drugs and macromolecular drugs have been poorly developed in supramolecular materials (Geng et al., 2020). Some macromolecular drugs, such as the monoclonal antibodies, which play a specifically anti-inflammatory role in CRS, might fail to be delivered by supramolecular materials due to their large sizes. Further research is necessary for new strategies to recognize proteins through heteromultivalency or macrocycles with much larger cavity sizes for protein delivery.

Traditional drug delivery systems have widely been used in many fields of biomedicine. Moving forward, we anticipate that more supramolecular materials can replace traditional materials as novel delivery platforms in clinical practice.

\section{REFERENCES}

Aletaha, D., and Smolen, J. S. (2018). Diagnosis and Management of Rheumatoid Arthritis. JAMA 320 (13), 1360-1372. doi:10.1001/jama.2018.13103

Bajpayee, A. G., and Grodzinsky, A. J. (2017). Cartilage-targeting Drug Delivery: Can Electrostatic Interactions Help?. Nat. Rev. Rheumatol. 13 (3), 183-193. doi:10.1038/nrrheum.2016.210

Barnes, P. J. (2011). Glucocorticosteroids: Current and Future Directions. Br. J. Pharmacol. 163 (1), 29-43. doi:10.1111/j.1476-5381.2010.01199.x

Butterfield, S. M., Patel, P. R., and Waters, M. L. (2002). Contribution of Aromatic Interactions to $\alpha$-Helix Stability. J. Am. Chem. Soc. 124 (33), 9751-9755. doi:10.1021/ja026668q

Cassese, S., Byrne, R. A., Ndrepepa, G., Kufner, S., Wiebe, J., Repp, J., et al. (2016). Everolimus-eluting Bioresorbable Vascular Scaffolds versus EverolimusEluting Metallic Stents: a Meta-Analysis of Randomised Controlled Trials. The Lancet 387 (10018), 537-544. doi:10.1016/S0140-6736(15)00979-4

Chotiyarnwong, P., and McCloskey, E. V. (2020). Pathogenesis of GlucocorticoidInduced Osteoporosis and Options for Treatment. Nat. Rev. Endocrinol. 16 (8), 437-447. doi:10.1038/s41574-020-0341-0

Chung, C. H., Jung, W., Keum, H., Kim, T. W., and Jon, S. (2020). Nanoparticles Derived from the Natural Antioxidant Rosmarinic Acid Ameliorate Acute Inflammatory Bowel Disease. ACS Nano 14 (6), 6887-6896. doi:10.1021/ acsnano.0c01018

Deirram, N., Zhang, C., Kermaniyan, S. S., Johnston, A. P. R., and Such, G. K. (2019). pH-Responsive Polymer Nanoparticles for Drug Delivery. Macromol. Rapid Commun. 40 (10), 1800917. doi:10.1002/marc.201800917

Dünnhaupt, S., Kammona, O., Waldner, C., Kiparissides, C., and BernkopSchnürch, A. (2015). Nano-carrier Systems: Strategies to Overcome the Mucus Gel Barrier. Eur. J. Pharmaceutics Biopharmaceutics 96, 447-453. doi:10.1016/j.ejpb.2015.01.022

Feldblum, E. S., and Arkin, I. T. (2014). Strength of a Bifurcated H Bond. Proc. Natl. Acad. Sci. 111 (11), 4085-4090. doi:10.1073/pnas.1319827111

Fokkens, W., Desrosiers, M., Harvey, R., Hopkins, C., Mullol, J., Philpott, C., et al. (2019). EPOS2020: Development Strategy and Goals for the Latest European Position Paper on Rhinosinusitis. Rhin 57 (3), 162-169. doi:10.4193/ Rhin 17.25310.4193/rhin 19.080

Fraile, J. M., Garcia-Martin, E., Gil, C., Mayoral, J. A., Pablo, L. E., Polo, V., et al. (2016). Laponite as Carrier for Controlled In Vitro Delivery of Dexamethasone in Vitreous Humor Models. Eur. J. Pharmaceutics Biopharmaceutics 108, 83-90. doi:10.1016/j.ejpb.2016.08.015

Geng, W.-C., Sessler, J. L., and Guo, D.-S. (2020). Supramolecular Prodrugs Based on Host-Guest Interactions. Chem. Soc. Rev. 49 (8), 2303-2315. doi:10.1039/c9cs00622b

Guo, H., Xu, W., Chen, J., Yan, L., Ding, J., Hou, Y., et al. (2017). Positively Charged Polypeptide Nanogel Enhances Mucoadhesion and Penetrability of 10-

\section{AUTHOR CONTRIBUTIONS}

$\mathrm{YH}, \mathrm{FZ}, \mathrm{SM}, \mathrm{JZ}, \mathrm{XW}, \mathrm{YZ}$, and LZ discussed and wrote the manuscript.

\section{FUNDING}

This work was supported by grants from the national key R and D program of China (No 2016YFC0905200), the program for the Changjiang scholars and innovative research team (IRT13082), the national natural science foundation of China (Nos 81630023, 81970852, and 82000962), the Beijing Bai-Qian-Wan talent project (2019A32), the Public Welfare Development and Reform Pilot Project (2019-10), the National Science and Technology Major Project (2019ZX09201-005) and the CAMS Innovation Fund for Medical Sciences (2019-I2M-5-022).

hydroxycamptothecin in Orthotopic Bladder Carcinoma. J. Controlled Release 259, 136-148. doi:10.1016/j.jconrel.2016.12.041

Han, J. K., Forwith, K. D., Smith, T. L., Kern, R. C., Brown, W. J., Miller, S. K., et al. (2014). RESOLVE: a Randomized, Controlled, Blinded Study of Bioabsorbable Steroid-Eluting Sinus Implants for In-Office Treatment of Recurrent Sinonasal Polyposis. Int. Forum Allergy Rhinol. 4 (11), 861-870. doi:10.1002/alr.21426

Hopkins, C. (2019). Chronic Rhinosinusitis with Nasal Polyps. N. Engl. J. Med. 381 (1), 55-63. doi:10.1056/NEJMcp1800215

Janisiewicz, A., and Lee, J. T. (2015). In-office Use of a Steroid-Eluting Implant for Maintenance of Frontal Ostial Patency after Revision Sinus Surgery. Allergy Rhinol (Providence) 6 (1), 68-75. doi:10.2500/ar.2015.6.0104

Jung, H. S., Lee, T., Kwon, I. K., Kim, H. S., Hahn, S. K., and Lee, C. S. (2015). Surface Modification of Multipass Caliber-Rolled $\mathrm{Ti}$ alloy with Dexamethasone-Loaded Graphene for Dental Applications. ACS Appl. Mater. Inter. 7 (18), 9598-9607. doi:10.1021/acsami.5b03431

Kern, R. C., Stolovitzky, J. P., Silvers, S. L., Singh, A., Lee, J. T., Yen, D. M., et al. (2018). A Phase 3 Trial of Mometasone Furoate Sinus Implants for Chronic Sinusitis with Recurrent Nasal Polyps. Int. Forum Allergy Rhinol. 8 (4), 471-481. doi:10.1002/alr.22084

Kim, B.-S., Park, S. W., and Hammond, P. T. (2008). Hydrogen-bonding Layer-ByLayer-Assembled Biodegradable Polymeric Micelles as Drug Delivery Vehicles from Surfaces. ACS Nano 2 (2), 386-392. doi:10.1021/nn700408z

Kohanski, M. A., Toskala, E., and Kennedy, D. W. (2018). Evolution in the Surgical Management of Chronic Rhinosinusitis: Current Indications and Pitfalls. J. Allergy Clin. Immunol. 141 (5), 1561-1569. doi:10.1016/j.jaci.2018.03.003

Lee, W. C., Lim, C. H. Y. X., Shi, H., Tang, L. A. L., Wang, Y., Lim, C. T., et al. (2011). Origin of Enhanced Stem Cell Growth and Differentiation on Graphene and Graphene Oxide. ACS Nano 5 (9), 7334-7341. doi:10.1021/nn202190c

Lühder, F., and Reichardt, H. (2017). Novel Drug Delivery Systems Tailored for Improved Administration of Glucocorticoids. Ijms 18 (9), 1836. doi:10.3390/ ijms 18091836

Ma, B., Xu, H., Zhuang, W., Wang, Y., Li, G., and Wang, Y. (2020). Reactive Oxygen Species Responsive Theranostic Nanoplatform for Two-Photon Aggregation-Induced Emission Imaging and Therapy of Acute and Chronic Inflammation. ACS Nano 14 (5), 5862-5873. doi:10.1021/acsnano.0c01012

Mayba, J. N., and Gooderham, M. J. (2017). Review of Atopic Dermatitis and Topical Therapies. J. Cutan. Med. Surg. 21 (3), 227-236. doi:10.1177/ 1203475416685077

Mendes, A. C., Baran, E. T., Reis, R. L., and Azevedo, H. S. (2013). Self-assembly in Nature: Using the Principles of Nature to Create Complex Nanobiomaterials. WIREs Nanomed Nanobiotechnol 5 (6), 582-612. doi:10.1002/wnan.1238

Murr, A. H., Smith, T. L., Hwang, P. H., Bhattacharyya, N., Lanier, B. J., Stambaugh, J. W., et al. (2011). Safety and Efficacy of a Novel Bioabsorbable, Steroid-Eluting Sinus Stent. Int. Forum Allergy Rhinology 1 (1), 23-32. doi:10.1002/alr.20020 
Nikoubashman, O., Heringer, S., Feher, K., Brockmann, M.-A., Sellhaus, B., Dreser, A., et al. (2018). Development of a Polymer-Based Biodegradable Neurovascular Stent Prototype: A Preliminary In Vitro and In Vivo Study. Macromol. Biosci. 18 (7), 1700292. doi:10.1002/mabi.201700292

Ogawa, Y., Kobayashi, T., Nishioka, A., Kariya, S., Ohnishi, T., Hamasato, S., et al. (2004). Reactive Oxygen Species-Producing Site in Hydrogen PeroxideInduced Apoptosis of Human Peripheral T Cells: Involvement of Lysosomal Membrane Destabilization. Int. J. Mol. Med. 13 (3), 383-388.

Raza, A., Hayat, U., Rasheed, T., Bilal, M., and Iqbal, H. M. N. (2018). Redoxresponsive Nano-Carriers as Tumor-Targeted Drug Delivery Systems. Eur. J. Med. Chem. 157, 705-715. doi:10.1016/j.ejmech.2018.08.034

Rykowska, I., Nowak, I., and Nowak, R. (2020). Drug-Eluting Stents and BalloonsMaterials, Structure Designs, and Coating Techniques: A Review. Molecules 25 (20), 4624. doi:10.3390/molecules 25204624

Safwat, M. A., Mansour, H. F., Hussein, A. K., Abdelwahab, S., and Soliman, G. M. (2020). Polymeric Micelles for the Ocular Delivery of Triamcinolone Acetonide: Preparation and In Vivo Evaluation in a Rabbit Ocular Inflammatory Model. Drug Deliv. 27 (1), 1115-1124. doi:10.1080/10717544.2020.1797241

Schneider, H.-J. (2009). Binding Mechanisms in Supramolecular Complexes. Angew. Chem. Int. Ed. 48 (22), 3924-3977. doi:10.1002/anie.200802947

Shen, Z.-l., Xia, Y.-q., Yang, Q.-s., Tian, W.-d., Chen, K., and Ma, Y.-q. (2017). Polymer-Nucleic Acid Interactions. Top. Curr. Chem. (Z) 375 (2), 44. doi:10.1007/s41061-017-0131-x

Shigemitsu, H., Kubota, R., Nakamura, K., Matsuzaki, T., Minami, S., Aoyama, T., et al. (2020). Protein-responsive Protein Release of Supramolecular/polymer Hydrogel Composite Integrating Enzyme Activation Systems. Nat. Commun. 11 (1), 3859. doi:10.1038/s41467-020-17698-0

Shim, G., Kim, M.-G., Kim, D., Park, J. Y., and Oh, Y.-K. (2017). Nanoformulationbased Sequential Combination Cancer Therapy. Adv. Drug Deliv. Rev. 115, 57-81. doi:10.1016/j.addr.2017.04.003

Shim, G., Kim, M.-G., Park, J. Y., and Oh, Y.-K. (2016). Graphene-based Nanosheets for Delivery of Chemotherapeutics and Biological Drugs. Adv. Drug Deliv. Rev. 105 (Pt B), 205-227. doi:10.1016/j.addr.2016.04.004

Sinnokrot, M. O., Valeev, E. F., and Sherrill, C. D. (2002). Estimates of the Ab Initio Limit for $\pi-\pi$ Interactions: The Benzene Dimer. J. Am. Chem. Soc. 124 (36), 10887-10893. doi:10.1021/ja025896h

Smith, T. L., Singh, A., Luong, A., Ow, R. A., Shotts, S. D., Sautter, N. B., et al. (2016). Randomized Controlled Trial of a Bioabsorbable Steroid-Releasing Implant in the Frontal Sinus Opening. The Laryngoscope 126 (12), 2659-2664. doi:10.1002/lary.26140

Tu, G.-w., Shi, Y., Zheng, Y.-j., Ju, M.-j., He, H.-y., Ma, G.-g., et al. (2017). Glucocorticoid Attenuates Acute Lung Injury through Induction of Type 2 Macrophage. J. Transl Med. 15 (1), 181. doi:10.1186/s12967-017-1284-7

van der Sluis, R. J., and Hoekstra, M. (2020). Glucocorticoids Are Active Players and Therapeutic Targets in Atherosclerotic Cardiovascular Disease. Mol. Cell Endocrinol. 504, 110728. doi:10.1016/j.mce.2020.110728

Vandewalle, J., Luypaert, A., De Bosscher, K., and Libert, C. (2018). Therapeutic Mechanisms of Glucocorticoids. Trends Endocrinol. Metab. 29 (1), 42-54. doi:10.1016/j.tem.2017.10.010

Wang, C., Wang, Z., and Zhang, X. (2012). Amphiphilic Building Blocks for SelfAssembly: from Amphiphiles to Supra-amphiphiles. Acc. Chem. Res. 45 (4), 608-618. doi:10.1021/ar200226d
Wang, Y., Liu, D., Zheng, Q., Zhao, Q., Zhang, H., Ma, Y., et al. (2014). Disulfide Bond Bridge Insertion Turns Hydrophobic Anticancer Prodrugs into Self-Assembled Nanomedicines. Nano Lett. 14 (10), 5577-5583. doi:10.1021/nl502044x

Wheatley, L. M., and Togias, A. (2015). Allergic Rhinitis. N. Engl. J. Med. 372 (5), 456-463. doi:10.1056/NEJMcp1412282

Wheeler, S. E. (2013). Understanding Substituent Effects in Noncovalent Interactions Involving Aromatic Rings. Acc. Chem. Res. 46 (4), 1029-1038. doi:10.1021/ar300109n

Wu, W., Zhang, Z., Xiong, T., Zhao, W., Jiang, R., Chen, H., et al. (2017). Calcium Ion Coordinated Dexamethasone Supramolecular Hydrogel as Therapeutic Alternative for Control of Non-infectious Uveitis. Acta Biomater. 61, 157-168. doi:10.1016/j.actbio.2017.05.024

Yadav, S., Sharma, A. K., and Kumar, P. (2020). Nanoscale Self-Assembly for Therapeutic Delivery. Front. Bioeng. Biotechnol. 8, 127. doi:10.3389/fbioe.2020.00127

Yu, L., Zhang, W., Luo, W., Dupont, R. L., Xu, Y., Wang, Y., et al. (2020). Molecular Recognition of Human Islet Amyloid Polypeptide Assembly by Selective Oligomerization of Thioflavin T. Sci. Adv. 6 (32), eabc1449. doi:10.1126/ sciadv.abc1449

Zavgorodnya, O., Carmona-Moran, C. A., Kozlovskaya, V., Liu, F., Wick, T. M., and Kharlampieva, E. (2017). Temperature-responsive Nanogel Multilayers of poly(N-Vinylcaprolactam) for Topical Drug Delivery. J. Colloid Interf. Sci. 506, 589-602. doi:10.1016/j.jcis.2017.07.084

Zhang, W., Mo, S., Liu, M., Liu, L., Yu, L., and Wang, C. (2020a). Rationally Designed Protein Building Blocks for Programmable Hierarchical Architectures. Front. Chem. 8, 587975. doi:10.3389/fchem.2020.587975

Zhang, W., Yu, L., Ji, T., and Wang, C. (2020b). Tumor MicroenvironmentResponsive Peptide-Based Supramolecular Drug Delivery System. Front. Chem. 8, 549. doi:10.3389/fchem.2020.00549

Zhou, J., Li, J., Du, X., and Xu, B. (2017). Supramolecular Biofunctional Materials. Biomaterials 129, 1-27. doi:10.1016/j.biomaterials.2017.03.014

Zhou, Y., Lei, L., Zhang, Z., Zhang, R., Song, Q., and Li, X. (2018). Cation Instructed Steroidal Prodrug Supramolecular Hydrogel. J. Colloid Interf. Sci. 528, 10-17. doi:10.1016/j.jcis.2018.05.059

Zhuang, W.-R., Wang, Y., Cui, P.-F., Xing, L., Lee, J., Kim, D., et al. (2019). Applications of $\pi-\pi$ Stacking Interactions in the Design of Drug-Delivery Systems. J. Controlled Release 294, 311-326. doi:10.1016/j.jconrel.2018.12.014

Conflict of Interest: The authors declare that the research was conducted in the absence of any commercial or financial relationships that could be construed as a potential conflict of interest.

Publisher's Note: All claims expressed in this article are solely those of the authors and do not necessarily represent those of their affiliated organizations, or those of the publisher, the editors and the reviewers. Any product that may be evaluated in this article, or claim that may be made by its manufacturer, is not guaranteed or endorsed by the publisher.

Copyright (c) 2021 Hao, Zhang, Mo, Zhao, Wang, Zhao and Zhang. This is an openaccess article distributed under the terms of the Creative Commons Attribution License (CC BY). The use, distribution or reproduction in other forums is permitted, provided the original author(s) and the copyright owner(s) are credited and that the original publication in this journal is cited, in accordance with accepted academic practice. No use, distribution or reproduction is permitted which does not comply with these terms. 